\title{
Evaluation of protection against squalene peroxidation with a combination of active compounds
}

Baba Teme ${ }^{1}$, Benoit Cadars ${ }^{1}$, Isabelle Benoit ${ }^{1}$.

${ }^{1}$ NAOS, France.

\section{INTRODUCTION}

Dysseborrhea is a modification of the qualitative composition of sebum that is thought to be one of the causes of acne. The peroxidation of squalene, a lipid present in up to $15 \%$ of human sebum, plays a large role in this process. Squalene peroxide makes sebum more viscous and comedogenic. For this reason, we decided to test whether or not active ingredients could effectively prevent the oxidation of squalene and be used in dermocosmetics for acne-prone skin.

\section{MATERIAL \& METHODS}

For this experiment we irradiated and stirred squalene for 80 hours (over 5 days) under a $500 \mathrm{~W}$ halogen spotlight placed $20 \mathrm{~cm}$ away to induce oxidation. Every day of the experiment we treated the squalene with active ingredients dissolved in DMSO. Solvent control was also realized. At the end of the protocol, squalene oxidation was assessed visually in terms of colour and viscosity. Viscosity was objectively measured with rheometer Anton Paar MCR102 and squalene degredation by peroxidation was measured with HPLC. The active compounds tested consisted of a mix of Bakuchiol, Ginkgo biloba extract, Mannitol and Propyl Gallate.

\section{RESULTS}

Non-irradiated squalene appears fluid, colourless, and translucent. After 80 hours of irradiation, the squalene became very viscous and yellow but still translucent. When protected with the active compounds mix, the squalene was fluid, pale yellow, and slightly cloudy (Fig.1).

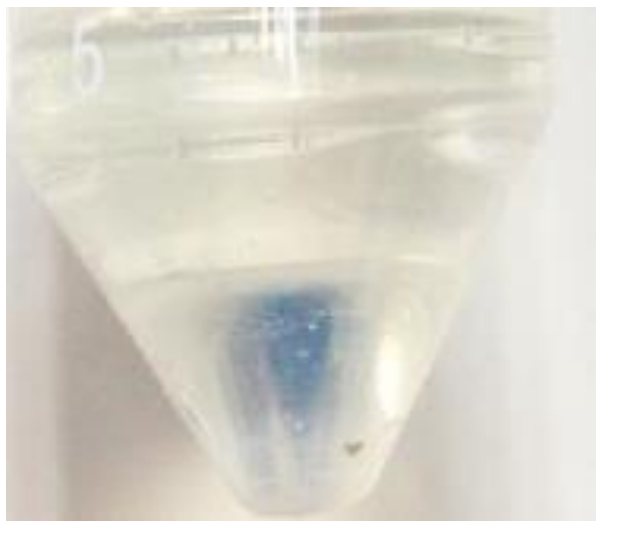

Unirradiated Untreated

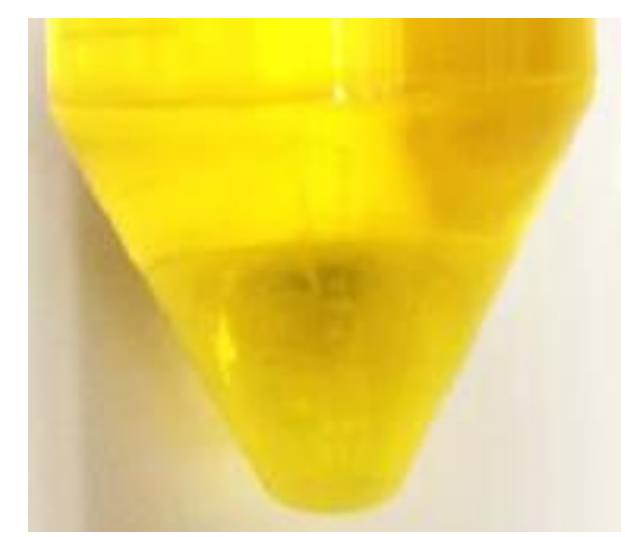

Irradiated Untreated

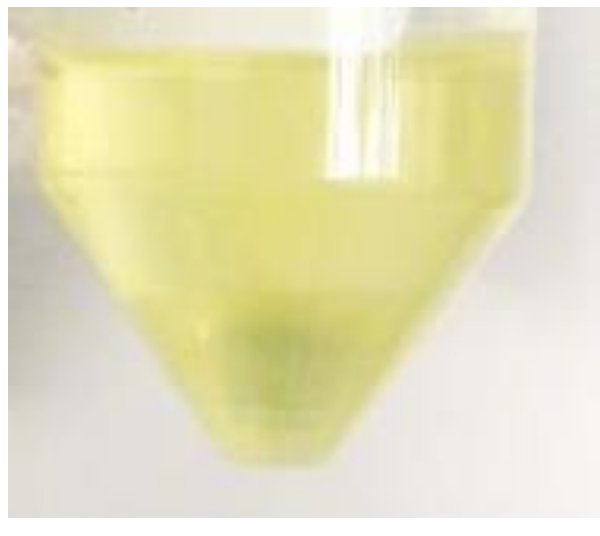

Irradiated Treated

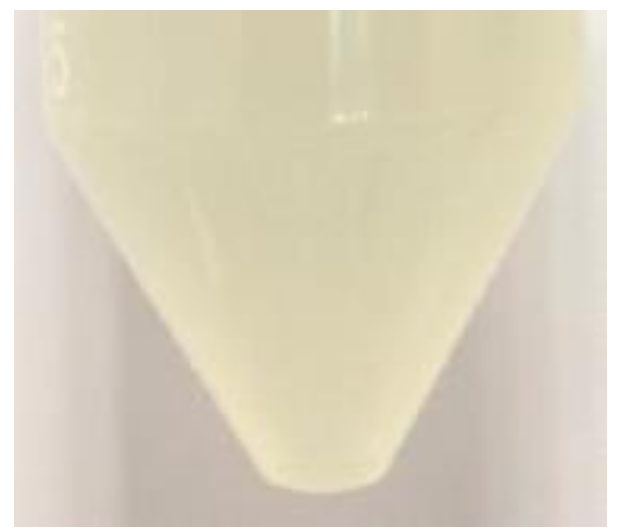

Unirradiated Treated

Fig. 1 Visual assessment of samples

The irradiation of untreated squalene led to a significant degradation of squalene through peroxidation (Fig.2).

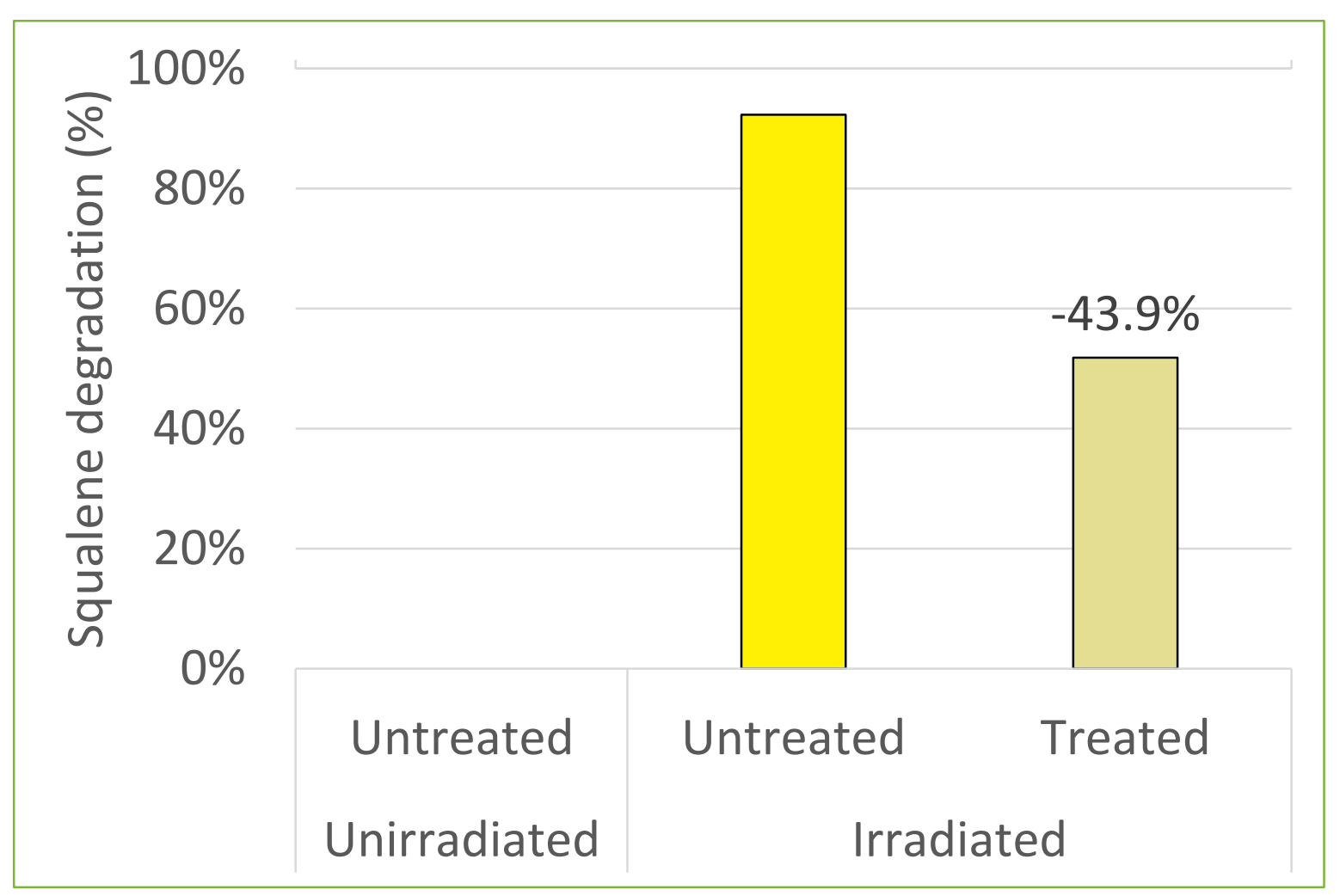

Fig.2 Protection against squalene oxidation
The treatment of squalene with the selected active compounds during oxidation led to a 43.9 decrease of its degradation (Fig.3).

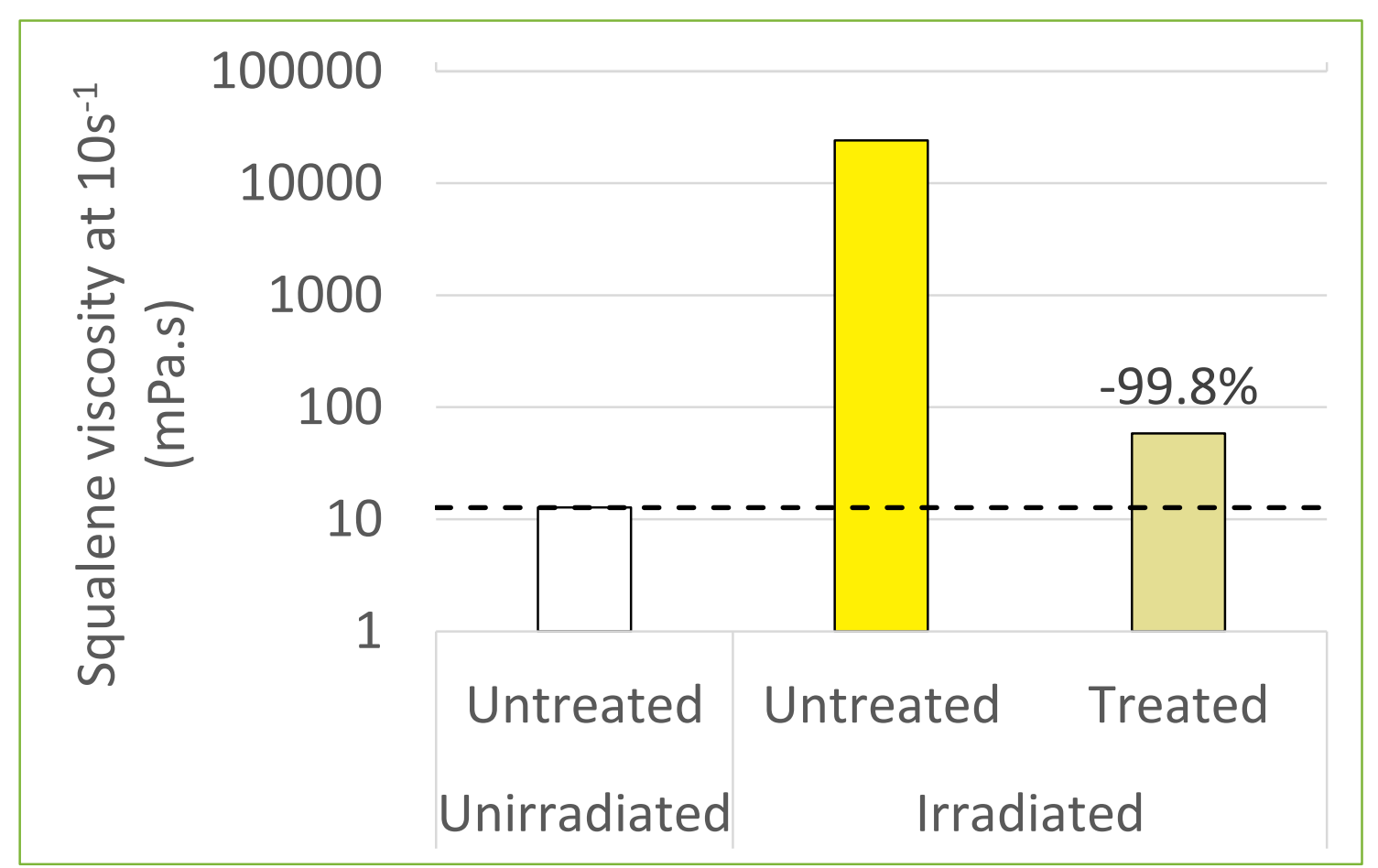

Fig.3 Protection against squalene oxidation-induced thickening

\section{CONCLUSION}

The association of Bakuchiol, Ginkgo biloba extract, Mannitol and Propyl Gallate effectively protected squalene against peroxidation and subsequent thickening, which are parts of dysseborrhea. This experiment demonstrates that these active compounds are well suited for dermocosmetics designed for acne-prone skin, whose objective is to counterbalance dysseborrhea. 